\title{
Transient mutism following neuroendoscopy in children; report of two cases
}

\author{
Nima Derakhshan* and Mohammad Sadegh Masoudi
}

\begin{abstract}
Background: Since the introduction of neuro-endoscopy by Walter Dandy and his colleagues in the early 1920's, several efforts has been made to improve this tool for treatment of hydrocephalus, brain tumors, arachnoid cysts, etc and has largely substituted craniotomy procedures. Several complications such as CSF leakage, intraventicular hemorrhage, infectious complications and transient and permanent psycho-neurologic deficits have been reported for neuro-endoscopic procedures in children. Here in, two cases of transient mutism following neuroendoscopy in children were reported, with special focus on pathophysiology, management and clinical course. Knowledge about the possible complications of a procedures, empowers the pediatric neurosurgeon with the ability to avoid them and also to successfully handle them once they take place.

Case presentation: We report two patients who underwent neuro-endoscopy,first for treatment of arachnoid cyst via cystoventriculostomy and second for endoscopic third ventriculostomy(ETV) to treat hydrocephalus. Both children developed apathy and mutism following the procedure. However, this condition was transient and both children improved after a course of 5-6 days with conservative management.

Conclusions: Mutism following neuro-endoscopic procedures in children may result from excessive traction on paraventricular white matter and disruption of fronto-subcortical circuits. It can be avoided with less intra-procedural dilation of venricles and less traction on white matter tracts with choosing a more accurate trajectory. However, this condition is transient and spontaneous resolution wil ensue in several days wth conservative management.
\end{abstract}

\section{Case presentation}

\section{Case 1}

A 3 month old boy was referred to pediatric neurosurgery clinic due to increased head circumference. He was a product of cesarean section to a nulliparous mother after an uneventful full-term pregnancy and born with an uneventful delivery. His head circumference was $37 \mathrm{~cm}$ at birth and $44 \mathrm{~cm}$ when he was visited at the clinic. Diameters of his bulged anterior fontanel were $4 * 6 \mathrm{~cm}$. His brain MRI revealed a huge quadrigeminal cistern arachnoid cyst which walls was bulged into right lateral ventricle.

Through a standard burrhole on right Kocher's point, a rigid neuroendoscope (Karl Storz GmBH, Tottingen, Germany) with a $1.3 \mathrm{~mm}$ diameter working channel was introduced into the right lateral ventricle and cyst wall

\footnotetext{
*Correspondence: nima_med83@yahoo.com

Shiraz Neuroscience Research Center, Department of Neurosurgery, Shiraz University of Medical Sciences, Shiraz, Iran

was punctured and opened to lateral ventricle via grasping forceps with double action jaws, $1.3 \mathrm{~mm}$ diameter and working length of $30 \mathrm{~cm}$.

Post-operatively the patient's vital signs were within normal ranges for his age, he was afebrile and there was no evidence of hemorrhage in his brain computedtomography scan. Although his primitive reflexes of grasp, Moro and sucking was normal, he was mute and apathic to the environment. His mother noted that he didn't cry due to hunger and also after tapping on his heels, unlike the pre-operative course. During the 6th post-operative day, he cried due to hunger and his mother noted that his infant had become more attentive to the environment.

\section{Case 2}

A 6 month old boy was consulted from pediatric service due to ventriculo-peritoneal (VP) shunt malfunction. He was a case of congenital hydrocephalus due to aqueduct 
stenosis that underwent right anterior VP shunt when he was 27 days old. He had recently developed sun-setting of eyes and his head circumference was increased from 40 to 45 during the last month. His anterior fontanel was bulged and tense with diameters of $5 * 6 \mathrm{~cm}$. The shunt pump didn't function appropriately; so with diagnosis of shunt malfunction, we removed his VP shunt and through the same route, a rigid neuroendoscope (Karl Storz $\mathrm{GmBH}$, Tottingen, Germany) with a $1.3 \mathrm{~mm}$ diameter working channel was introduced into the right lateral ventricle. Endoscopic third ventriculostomy was performed using a grasping forceps with double action jaws, $1.3 \mathrm{~mm}$ diameter and working length of $30 \mathrm{~cm}$ by opening of tuber cinerum to basal cisterns. CSF flow was seen at the stoma and the procedure was successful. Post-operatively the patient's vital signs were within normal ranges for his age and he was afebrile. His sun-setting eyes had not much improved but his fontanel was not bulged anymore. His mother noted that he doesn't cry due to hunger as he did before, but his breast feeding was normal. On physical examination his Moro and sucking reflexes were good but the child seemed mute and apathic and didn't cry after tapping his heels.

He gradually started to babble and make gurgling sounds after 5-6days and cried in response to painful stimuli.

\section{Conclusion}

Neuroendoscopy is replacing traditional craniotomy and shunting procedures, worldwide for management of hydrocephalus, brain tumors, arachnoid cysts and etc.

Several complications are mentioned for neuroendoscopic procedures for children in the literature, which include hemorrhage (intraventricular, intraparenchymal, subdural, epidural and subarachnoid), CSF leakage, CSF infections, vascular insults (basilar rupture, thalamic infarcts), hypothalamic injury, memory disturbance, focal neurologic deficits and delayed sudden death $[1,2]$. Although the rate of transient morbidities is about $8 \%[3,4]$, only $2 \%$ will have permanent morbidity and mortality rate of about $0.2 \%$ is reported in different series $[3,4]$.

In a 2007 review by Cinalli and coworkers [5], transient akinetic mutism was seen in 1 of their 231 patients who underwent neuroendoscopic procedures. This patient suddenly passed away due to unexplained events (probably due to acute hydrocephalus following closure of stoma) 6 months after the procedure and was the only mortality presented according to their report.

To my knowledge this is the first report of two cases with transient mutism, apathy and inability to cry following neuroendoscopic procedures in children. Both cases improved after a course of 5-6 days. The pathogenesis of mutism in these cases is not well understood. However, knowledge that this phenomenon is transient is necessary and will let the pediatric neurosurgeon reassure the parents that the child will regain his pre-operative status of attention to the environment and speech milestones.

This complication may result from excessive traction on paraventricular white matter [6] and disruption of fronto-subcortical circuits [7]. This complication can be avoided with minimizing the manipulation of neural structures and avoiding repeated dilatation of ventricles during the procedure. Most of these complications are temporary and do not mandate a surgical intervention. If the symptoms persist or aggravated during the few post-operative days, dopaminergic agents may prove useful in alleviating these symptoms.

No surgery is risk-free; however, a thorough understanding of limitations and possible complications associated with a specific surgical procedure, will help the surgeon to avoid them or better manage them if they are inevitable.

\section{Abbreviations}

CSF, cerebrospinal fluid; ETV, endoscopic third ventriculostomy; $V P$, ventriculo-peritoneal

\section{Acknowledgement}

None

\section{Funding}

No funding sources were available in any parts of this work.

\section{Authors' contribution}

ND carried out the designing the concept and writing all sections of this manuscript and has read and approved this manuscript. MSM supervised the revisions requested by the reviewers. Both authors read and approved the final manuscript.

\section{Competing interests}

There isn't any conflict of interest to be declared regarding the manuscript

\section{Consent for publication}

Consent for the publication of these case reports was obtained from the legally authorized representatives (parents) of the two children.

Ethics approval and consent to participate

Not applicable; however the parents of both children gave informed consent for the neuroendoscopic surgery

Received: 9 September 2015 Accepted: 31 May 2016

Published online: 18 July 2016

References

1. Bouras T, Sgouros S. Complications of endoscopic third ventriculostomy. J Neurosurg Pediatr. 2011;7(6):643-9.

2. Bouras T, Sgouros S. Complications of endoscopic third ventriculostomy: a systematic review. Acta Neurochir Suppl. 2012;113:149-53.

3. Bouras T, Sgouros S. Complications of endoscopic third ventriculostomy. World Neurosurg. 2013;79(2 Suppl):S22.e9-12.

4. Schroeder HW, Niendorf WR, Gaab MR. Complications of endoscopic third ventriculostomy. J Neurosurg. 2002;96(6):1032-40.

5. Cinalli G, Spennato P, Ruggiero C, Aliberti F, Trischitta V, Buonocore MC, Cianciulli E, Maggi G. Complications following endoscopic intracranial procedures in children. Childs Nerv Syst. 2007;23(6):633-44.

6. Akiyama T, Tanizaki Y, Akaji K, Hiraga K, Akiyama T, Takao M, Ohira T. Severe parkinsonism following endoscopic third ventriculostomy for non-communicating hydrocephalus-case report. Neurol Med Chir (Tokyo) 2011:51(1):60-3.

7. Grunsfeld AA, Login IS. Abulia following penetrating brain injury during endoscopic sinus surgery with disruption of the anterior cingulate circuit: case report. BMC Neurol. 2006;6:4. 